Artículo

\title{
Adaptación de genotipos de frijol negro a diferentes ambientes de Veracruz y Chiapas
}

\author{
Oscar Hugo Tosquy-Valle ${ }^{1}$ \\ Bernardo Villar-Sánchez ${ }^{2}$ \\ José Raúl Rodríguez-Rodríguez ${ }^{3}$ \\ Francisco Javier Ibarra-Pérez ${ }^{1 \S}$ \\ Rigoberto Zetina-Lezama ${ }^{1}$ \\ Pablo Andrés-Meza ${ }^{4}$ \\ José Luís Anaya López
}

${ }^{1}$ Campo Experimental Cotaxtla-INIFAP. Carretera Veracruz-Córdoba km 34.5, Medellín de Bravo, Veracruz, México. CP. 91700. (tosquy.oscar@inifap.gob.mx; zetina.rigoberto@inifap.gob.mx). ${ }^{2}$ Campo Experimental Centro de Chiapas-INIFAP. Carretera Ocozocoautla-Cintalapa km 3.0, Ocozocoautla, Chiapas. CP. 29140. (villar.bernardo@inifap.gob.mx). ${ }^{3}$ Campo Experimental Ixtacuaco-INIFAP. Carretera Martínez de la Torre-Tlapacoyan km 4.5, Martínez de la Torre, Veracruz. (rodriguez.jose@inifap.gob.mx). ${ }^{4}$ Facultad de Ciencias Agrícolas-Universidad Veracruzana-Campus Peñuela. Carretera Peñuela-Amatlán km 177, Córdoba, Veracruz. (pandres272@gmail.com). ${ }^{5}$ Campo Experimental Bajío-INIFAP. Carretera Celaya-San Miguel de Allende km 6.5, Celaya, Guanajuato. CP. 38000. (anaya.jose@inifap.gob.mx).

${ }^{\S}$ Autor para correspondencia: ibarra.francisco@ inifap.gob.mx.

\section{Resumen}

En la presente investigación se utilizó el modelo efectos principales aditivos e interacción multiplicativa (AMMI) para determinar el rendimiento y estabilidad de 12 líneas y dos variedades de frijol negro opaco, evaluadas durante 2016 y 2017, en 10 ambientes de Veracruz y Chiapas, México. El ensayo se estableció en diseño experimental bloques al azar con tres repeticiones y parcelas de tres surcos de $5 \mathrm{~m}$ de longitud. Se cuantificó el rendimiento de grano, el cual se analizó de manera individual por ambiente y combinada (ambientes-genotipos) de los 10 ambientes de prueba, también se estimaron los parámetros de estabilidad con el modelo AMMI. El Campo Experimental Ixtacuaco y Rincón Grande, Veracruz. en otoño-invierno de 2016-2017 bajo humedad residual, fueron los ambientes que combinaron baja interacción y alta productividad, por lo que son ideales para identificar genotipos con alto y estable rendimiento. Venustiano Carranza, Chiapas, en otoño-invierno de 2016-2017, El Rubí, Veracruz, en invierno-primavera de 2017 con riego y Nuevo México, Chiapas, en verano de 2016, en suelo ácido encalado, fueron los ambientes que más interaccionaron con los genotipos. La línea Jamapa Plus/XRAV-187-3-1-8, mostró la menor interacción con el ambiente (muy superior a las variedades Negro Comapa y Negro Grijalva), así como alto rendimiento (1 $437.3 \mathrm{~kg} \mathrm{ha}^{-1}$ ), mientras que, Jamapa Plus/XRAV-187-3$1-2$, fue la línea más rendidora (1504.3 $\mathrm{kg} \mathrm{ha}^{-1}$ ), pero su adaptación fue específica, en los ambientes con estrés por acidez edáfica en el centro de Chiapas y por sequía terminal en el centro de Veracruz.

Palabras clave: Phaseolus vulgaris L., genotipo-ambiente, interacción, líneas mejoradas.

Recibido: mayo de 2019

Aceptado: agosto de 2019 


\section{Introducción}

En Veracruz y Chiapas, México, durante 2016 se sembraron 150784 ha de frijol de grano negro opaco, tipo tropical (SIAP, 2018), que es el de mayor demanda comercial en la región del sureste de México, en donde se produce el 37.3\% de esta clase de frijol en el país (Rodríguez et al., 2010; FIRA, 2016). El rendimiento promedio en ambas entidades es bajo ( $<650 \mathrm{~kg} \mathrm{ha}^{-1}$ ) (SIAP, 2018), debido a que el cultivo es afectado por factores biótico y abióticos.

En los primeros destaca la incidencia de enfermedades como el mosaico amarillo dorado del frijol (BGYMV), el mosaico común del frijol (BCMV), la roya [Uromyces appendiculatus var. appendiculatus (Pers.) Unger] y la mancha angular [Pseudocercospora griseola (Sacc.) Ferraris] (López et al., 2006; Tosquy et al., 2012). Mientras que, en los abióticos, los más importantes son: la ocurrencia de sequía intra-estival (que comúnmente se presenta del 20 de julio al 20 de agosto), en las siembras de temporal del ciclo de verano y de sequía terminal, que frecuentemente ocurre después de la floración del cultivo, cuando el frijol se establece en condiciones de humedad residual, en el ciclo de otoño-invierno (Tosquy et al., 2017), así como la siembra de frijol en suelos de baja fertilidad, ácidos y con alta saturación de aluminio (Villar et al., 2003; Tosquy et al., 2008).

Para contribuir a solucionar la problemática indicada, en el Programa de Mejoramiento de Frijol del Campo Experimental Cotaxtla (CECOT) del Instituto Nacional de Investigaciones Forestales, Agrícolas y Pecuarias (INIFAP), se evalúan líneas de generaciones tempranas y avanzadas de frijol negro opaco en viveros de adaptación y en ensayos regionales de rendimiento, que se conducen en diferentes localidades, condiciones de humedad y ciclos agrícolas de cultivo, en el sureste de México, de tal manera, que permita identificar las más sobresalientes por su rendimiento, estabilidad y adaptabilidad y con características agronómicas superiores a las de las variedades utilizadas actualmente (López et al., 2012).

Para determinar la estabilidad del rendimiento de genotipos, en el Programa de Mejoramiento de Frijol del CECOT, se ha utilizado el modelo univariado propuesto por Eberhart y Russell (1966), quienes proponen como parámetros de estabilidad, el coeficiente de regresión (Bi) y la desviación de la regresión $\left(\mathrm{S}^{2} \mathrm{di}\right)$, por lo que una variedad se considera estable, cuando $\mathrm{Bi}=1$ y $\mathrm{S}^{2} \mathrm{di}=0$, en tanto que, otras consideraciones de valores para estos parámetros indican que los genotipos son inestables.

En los últimos años, se ha aplicado el modelo multivariado AMMI (additive main effects and multiplicative interaction) descrito por Crossa et al. (1990), por ser más adecuado para estimar la estabilidad, debido a que permite describir e interpretar los efectos de la IGA (Gauch Jr. y Furnas, 1991; Brancourt-Hulmel y Lecomte, 2003), además de que es más efectivo para caracterizar la respuesta de los genotipos en los ambientes (Williams et al., 2010; Vargas et al., 2016). Los resultados pueden graficarse en un biplot de doble entrada (biplot CP1 vs rendimiento), donde se colocan tanto los efectos principales, como los efectos de interacción, para los genotipos y los ambientes, lo que facilita la interpretación de la IGA (Vallejo, 2005; López et al., 2015). El objetivo del presente trabajo de investigación fue identificar líneas élite que superen en rendimiento y adaptación a diferentes ambientes de producción, a dos variedades que se cultivan en los estados de Veracruz y Chiapas. 


\section{Materiales y métodos}

El ensayo uniforme incluyó 12 líneas élite seleccionadas por su rendimiento, adaptación o tolerancia a uno o más de los factores limitantes: suelo ácido, sequía y enfermedades (Garrido et al., 2017; Ibarra et al., 2017), las cuales fueron generadas por el Programa Nacional de Frijol del INIFAP (cuatro provenientes de la cruza Papaloapan/SEN-46, cinco de la cruza Negro Citlali/XRAV-187-3 y tres de Jamapa Plus/XRAV-187-3). Como testigos se utilizaron las variedades Negro Grijalva y Negro Comapa, liberadas por el INIFAP para las áreas tropicales del sureste de México, por su alto potencial de rendimiento, amplia adaptación y tolerancia a enfermedades (Villar et al., 2009; López et al., 2012).

El ensayo se estableció en tres ambientes del centro del estado de Chiapas y siete ambientes del estado de Veracruz (cuatro en la zona centro, dos en la zona sur y uno en la norte). La ubicación de los sitios experimentales, el ciclo de siembra y la condición ambiental en la que se condujo el ensayo se muestra en el Cuadro 1.

Cuadro 1. Localización y características de sitios experimentales donde se condujo el ensayo uniforme regional de rendimiento en Veracruz y Chiapas.

\begin{tabular}{|c|c|c|c|c|c|}
\hline Localidad & Municipio/estado & Ciclo/año & Condición ambiental & $\begin{array}{l}\text { Localización } \\
\text { (LN y LO) }\end{array}$ & $\begin{array}{l}\text { Altitud } \\
(\mathrm{m})\end{array}$ \\
\hline Nuevo México & Villaflores, Chis. & V / 2016 & $\begin{array}{l}\text { T- Suelo ácido } \\
(\mathrm{pH}>5.6)-\mathrm{CD}\end{array}$ & $16^{\circ} 27^{\prime}$ y $93^{\circ} 26^{\prime}$ & 660 \\
\hline Nuevo México & Villaflores, Chis. & V / 2016 & T-Suelo ácido $(\mathrm{pH}<4.4)$ & $16^{\circ} 27^{\prime}$ y $93^{\circ} 26^{\prime}$ & 660 \\
\hline Carranza & Ocozocoautla, Chis. & OI / 2016-17 & Humedad residual & $16^{\circ} 20^{\prime}$ y $92^{\circ} 35^{\prime}$ & 597 \\
\hline Rincón Gde. & Orizaba, Ver. & OI / 2016-17 & Humedad residual & $18^{\circ} 51^{\prime}$ y $97^{\circ} 06^{\prime}$ & 1248 \\
\hline El Rubí & Medellín, Ver. & OI / 2016-17 & Humedad residual & $18^{\circ} 55^{\prime}$ y $96^{\circ} 11^{\prime}$ & 22 \\
\hline ITA-JRC & Rodríguez Clara, Ver. & OI / 2016-17 & $\begin{array}{l}\text { HR-Suelo ácido (pH } \\
>6.1)-C D\end{array}$ & $18^{\circ} 01^{\prime}$ y $95^{\circ} 24^{\prime}$ & 133 \\
\hline ITA-JRC & Rodríguez Clara, Ver. & OI / 2016-17 & $\begin{array}{l}\text { HR-Suelo ácido (pH } \\
<4.7)\end{array}$ & $18^{\circ} 01^{\prime} y 95^{\circ} 24^{\prime}$ & 133 \\
\hline CEIXTA & Tlapacoyan, Ver. & OI / 2016-17 & Humedad residual & $20^{\circ} 02^{\prime}$ y $97^{\circ} 05^{\prime}$ & 88 \\
\hline El Rubí & Medellín, Ver. & IP / 2017 & Riego durante el ciclo & $18^{\circ} 55^{\prime}$ y $96^{\circ} 11^{\prime}$ & 22 \\
\hline El Rubí & Medellín, Ver. & IP / 2017 & Sequía terminal & $18^{\circ} 55^{\prime}$ y $96^{\circ} 11^{\prime}$ & 22 \\
\hline
\end{tabular}

ITA-JRC= Instituto Tecnológico Agropecuario de Juan Rodríguez Clara; CEIXTA= Campo Experimental Ixtacuaco; $\mathrm{V}=$ ciclo de verano; $\mathrm{OI}=$ ciclo de otoño-invierno; $\mathrm{IP}=$ ciclo de invierno-primavera; $\mathrm{T}=$ temporal. $\mathrm{HR}=$ humedad residual; $\mathrm{CD}=$ con aplicación de dolomita.

Los genotipos se sembraron a una densidad de 250000 plantas ha $^{-1}$, en diseño experimental bloques completos al azar con tres repeticiones y parcelas de tres surcos de $5 \mathrm{~m}$ de longitud, donde la parcela útil correspondió al surco central completo. Todos los genotipos son de hábito de crecimiento indeterminado, tipo II, de plantas arbustivas y erectas (Singh, 1982).

En uno de los dos ensayos de la localidad de Nuevo México, Chis., establecidos en el ciclo verano de 2016 y otro del Instituto Tecnológico de Juan Rodríguez Clara (ITA-JRC), Ver., en otoñoinvierno de 2016-17, antes de la siembra se aplicaron 2 y 2.5 t ha $^{-1}$ de cal dolomita al suelo, respectivamente, para alcanzar un $\mathrm{pH}$ que se encuentre dentro del rango óptimo de 5.5 a 7.5 y así 
obtener un adecuado desarrollo de las plantas de frijol (Arias et al., 2007; Ruiz et al., 2013), los otros dos ensayos establecidos en ambas localidades se condujeron bajo condiciones naturales de estrés por suelo ácido.

En El Rubí, Veracruz, en invierno-primavera de 2017, un ensayo se condujo con riego durante todo el ciclo fenológico del frijol y el otro con suspensión de riego, a partir de la etapa reproductiva del cultivo (sequía terminal). Cabe indicar, que durante la conducción de los ensayos de campo no hubo incidencia de enfermedades que afectarán el rendimiento de frijol, el cual se estimó en kilogramos por hectárea al $14 \%$ de humedad.

Los datos de rendimiento de grano se analizaron de manera individual y combinada (ambientesgenotipos) de los 10 ambientes de prueba. En los casos en que se detectó significancia, para la separación de promedios se aplicó la prueba basada en la diferencia mínima significativa (DMS, $\alpha=0.05)$. Asimismo, se utilizó el modelo de efectos principales aditivos e interacción multiplicativa (AMMI) por sus siglas en inglés, para clasificar los ambientes e identificar genotipos sobresalientes por su rendimiento de grano y menor interacción con el ambiente (Gauch y Zobel, 1996).

Para el análisis de varianza y los parámetros de estabilidad se utilizó el programa de computo SAS (SAS Institute, 1999) y en el desarrollo del análisis AMMI se siguieron las recomendaciones de Vargas y Crossa (2000).

\section{Resultados y discusión}

\section{Rendimiento de grano}

De acuerdo al análisis combinado, el rendimiento varió significativamente $(p \leq 0.01)$ entre ambientes, genotipos y en la interacción de ambos factores. El Cuadro 2 muestra que en Carranza, Chiapas, ciclo de otoño-invierno de 2016-2017, bajo condiciones de humedad residual (A3), se obtuvo mayor rendimiento promedio y fue significativamente superior al resto de ambientes.

Cuadro 2. Rendimiento de grano $\left(\mathrm{kg} \mathrm{ha}^{-1}\right)$ de genotipos de frijol negro evaluados en 10 ambientes de Veracruz y Chiapas, México. Ciclos de verano de 2016, otoño-invierno de 2016-2017 e invierno-primavera de 2017.

\begin{tabular}{clccccc}
\hline \multirow{2}{*}{$\mathrm{T}$} & \multicolumn{2}{c}{ Genotipo } & \multicolumn{5}{c}{ Ambientes de prueba } \\
\cline { 3 - 7 } & & A1 & A2 & A3 & A4 & A5 \\
\hline G1 & Papaloapan/SEN 46-3-7 & $2116^{*}$ & 1120 & 1379 & $2097^{*}$ & 1367 \\
G2 & Papaloapan/SEN 46-6-6 & 1403 & 1004 & 1449 & $1952^{*}$ & 1097 \\
G3 & Papaloapan/SEN 46-7-7 & $1973^{*}$ & 560 & 1538 & 1693 & 1518 \\
G4 & Papaloapan/SEN 46-7-11 & 1181 & 848 & $2357^{*}$ & $1980^{*}$ & $1713^{*}$ \\
G5 & N Citlali/XRAV-187-3-1-6 & 1905 & 1007 & $2096^{*}$ & 1238 & $1580^{*}$ \\
G6 & N Citlali/XRAV-187-3-1-8 & 1655 & 1068 & $2409^{*}$ & 1298 & $1742^{*}$ \\
G7 & N Citlali/XRAV-187-3-14-6 & 1748 & 1068 & 1872 & $1720^{*}$ & 1260 \\
G8 & N Citlali/XRAV-187-3-14-7 & 1401 & 568 & $2420^{*}$ & 1438 & $1548^{*}$ \\
G9 & N Citlali/XRAV-187-3-16-7 & 1343 & $1335^{*}$ & 1378 & 1368 & 1368 \\
G10 & Jamapa Plus/XRAV-187-3-1-8 & 1615 & 1231 & $2586^{*}$ & 1447 & 1460 \\
\hline
\end{tabular}




\begin{tabular}{|c|c|c|c|c|c|c|c|}
\hline \multirow{2}{*}{$\mathrm{T}$} & \multirow{2}{*}{ Genotipo } & \multicolumn{6}{|c|}{ Ambientes de prueba } \\
\hline & & A1 & $\mathrm{A} 2$ & A3 & A4 & A5 & \\
\hline G11 & Jamapa Plus/XRAV-187-3-1-2 & $2276^{*}$ & 1304 * & 1692 & 1505 & $1660^{*}$ & \\
\hline G12 & Jamapa PlusX/RAV-187-3-4-4 & 1144 & 1071 & $2159^{*}$ & 1563 & $1887^{*}$ & \\
\hline G13 & Negro Comapa & 1876 & 1484 * & $2387^{*}$ & 1480 & 1270 & \\
\hline \multirow[t]{6}{*}{ G14 } & Negro Grijalva & 1863 & 1324 * & 2038 & 1469 & 1450 & \\
\hline & Promedio & $1678 \mathrm{~b}$ & $1071 \mathrm{c}$ & $1983 \mathrm{a}$ & $1589 \mathrm{~b}$ & $1494 \mathrm{~b}$ & \\
\hline & ANVA & $* *$ & $* *$ & ** & $*$ & $* *$ & \\
\hline & $\mathrm{CV}(\%)$ & 12.52 & 13.67 & 15.10 & 19.60 & 14.35 & \\
\hline & DMS (0.05) & 352.8 & 245.7 & 502.7 & 522.8 & 360.0 & \\
\hline & & A6 & A7 & A8 & A9 & A10 & Promedio \\
\hline G1 & Papaloapan/SEN 46-3-7 & 598 & 482 & $1755^{*}$ & 1328 & 1094 & 1333.5 abcde \\
\hline $\mathrm{G} 2$ & Papaloapan/SEN 46-6-6 & 693 & 505 & $1645^{*}$ & 1146 & 729 & $1162.2 \mathrm{e}$ \\
\hline G3 & Papaloapan/SEN 46-7-7 & 653 & 394 & 1603 & 1276 & 820 & $1202.8 \mathrm{de}$ \\
\hline G4 & Papaloapan/SEN 46-7-11 & 582 & 396 & 1609 & 1203 & 594 & 1246.4 bcde \\
\hline G5 & N Citlali/XRAV-187-3-1-6 & 633 & 455 & $1729^{*}$ & 1302 & 1080 & 1302.6 abcde \\
\hline G6 & N Citlali/XRAV-187-3-1-8 & $911^{*}$ & $706^{*}$ & $1876^{*}$ & 1250 & 859 & 1377.5 abcde \\
\hline G7 & N Citlali/XRAV-187-3-14-6 & 627 & 442 & 1375 & 1031 & 573 & $1171.6 \mathrm{e}$ \\
\hline G8 & N Citlali/XRAV-187-3-14-7 & 689 & 480 & 1538 & 1297 & 838 & 1221.7 cde \\
\hline G9 & N Citlali/XRAV-187-3-16-7 & 576 & 369 & 1473 & 1318 & 922 & $1144.9 \mathrm{e}$ \\
\hline G10 & Jamapa Plus/XRAV-187-3-1-8 & 603 & 481 & $1775^{*}$ & $1974 *$ & 1203 & $1437.3 \mathrm{abcd}$ \\
\hline G11 & Jamapa Plus/XRAV-187-3-1-2 & 715 & 475 & 1609 & $2271^{*}$ & $1536^{*}$ & $1504.3 \mathrm{a}$ \\
\hline G12 & Jamapa PlusX/RAV-187-3-4-4 & 767 & 576 & $1759^{*}$ & 1703 & 963 & 1359.3 abcde \\
\hline G13 & Negro Comapa & 661 & 512 & 1444 & $1964^{*}$ & $1385^{*}$ & $1446.2 \mathrm{abc}$ \\
\hline \multirow[t]{5}{*}{ G14 } & Negro Grijalva & 639 & 459 & $1724^{*}$ & $2307^{*}$ & $1437^{*}$ & $1471 \mathrm{ab}$ \\
\hline & Promedio & $668 d$ & $481 \mathrm{~d}$ & $1637 \mathrm{~b}$ & $1526 \mathrm{~b}$ & $1002 \mathrm{c}$ & 1312.9 \\
\hline & ANVA & 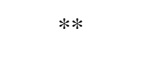 & & & & $* *$ & \\
\hline & $\mathrm{CV}(\%)$ & 11.08 & 11.77 & 8.75 & 20.04 & 16.29 & 16.36 \\
\hline & DMS (0.05) & 124.2 & 94.9 & 240.3 & 513.4 & 274.1 & 238.7 \\
\hline
\end{tabular}

$\mathrm{T}=$ tratamiento (genotipo); $\mathrm{G}=$ genotipo: $\mathrm{A}=$ ambiente; $\mathrm{A} 1=$ Nuevo México, Villaflores, Chiapas, con dolomita; $\mathrm{A} 2=$ Nuevo México, Villaflores, Chiapas, sin dolomita; A3= Carranza, Ocozocoautla, Chiapas; A4= Rincón Grande, Orizaba, Veracruz; A5=El Rubí, Medellín, Veracruz; A6= Rodríguez Clara, Ver., con dolomita; A7= Rodríguez Clara, Veracruz, sin dolomita; A8= CEIXTA, Tlapacoyan, Veracruz; A9= El Rubí, Medellín, Veracruz, riego; A10=El Rubí, Medellín, Veraruz, sequía; ${ }^{*}=$ genotipos estadísticamente superiores, según la diferencia mínima significativa (DMS, 0.05). Promedios de ambientes y genotipos con las mismas letras en la hilera y columna, respectivamente, son estadísticamente similares de acuerdo con la prueba de la DMS, 0.05 .

El mayor rendimiento promedio obtenido en esta localidad se debió principalmente a que el cultivo contó con humedad adecuada durante su ciclo de desarrollo (568 mm de precipitación pluvial en total), sin ocurrencia de periodos de sequía terminal. A su vez, en los ambientes de: Nuevo México, Chis., ciclo de verano de 2016, bajo condiciones de suelo ácido encalado (A1), CEIXTA, Ver. (A8) y Rincón Grande, Ver. (A4), ambos en otoño-invierno de 2016-2017 con humedad residual, así como en El Rubí, Ver., en invierno-primavera de 2017 bajo condiciones de riego (A9) y en otoñoinvierno de 2016-17 con humedad residual (A5), también se obtuvieron rendimientos promedio 
altos, porque en todos los casos, las condiciones de humedad para el desarrollo del frijol fueron adecuadas (más de $320 \mathrm{~mm}$ de agua durante el ciclo, de los cuales alrededor de $150 \mathrm{~mm}$ se proporcionaron mediante la aplicación de riegos o se recibieron por las lluvias que ocurrieron durante la etapa reproductiva del cultivo). Para un adecuado desarrollo y rendimiento del cultivo de frijol se requieren de al menos $300 \mathrm{~mm}$ de precipitación pluvial bien distribuidos durante su ciclo fenológico, siendo convenientes de 50 a $90 \mathrm{~mm}$, de la floración al llenado de vainas (Acosta et al., 2009; Ruiz et al., 2013).

Por el contrario, los rendimientos promedio más bajos se obtuvieron en el Instituto Tecnológico Agropecuario de Juan Rodríguez Clara (ITA-JRC), Veracruz, en otoño-invierno de 2016-17, en suelo ácido, con aplicación de cal dolomita (A6) y sin aplicación de dolomita (A7) (Cuadro 2). Lo anterior, fue como consecuencia principalmente del estrés hídrico que sufrieron las plantas de frijol durante su ciclo fenológico, ya que en el sitio experimental en donde se condujeron ambos ensayos, se tuvo una precipitación pluvial de la siembra hasta la cosecha de $150.4 \mathrm{~mm}$, de los cuales sólo $24.4 \mathrm{~mm}$ precipitaron durante la fase reproductiva del cultivo, específicamente de las etapas de formación de vainas al llenado de éstas, lo cual limitó el desarrollo de los genotipos en ambas condiciones de acidez de suelo. La falta de humedad durante la floración, la formación de vainas y el llenado de éstas, provoca una disminución significativa del rendimiento de grano, por una reducción en el número de vainas por planta y un deficiente llenado de vainas (Acosta et al., 2009).

En el mismo cuadro se muestra que; a través, de los ambientes de evaluación, Jamapa Plus/XRAV187-3-1-2, fue la línea más productiva, cuyo rendimiento de grano promedio fue estadísticamente similar al de otras cinco líneas y las variedades testigo, Negro Comapa y Negro Grijalva. Esta misma línea, junto con Negro Citlali/XRAV-187-3-1-8 obtuvo un rendimiento de grano significativamente sobresaliente en cinco de los 10 ambientes de prueba. Negro Citlali/XRAV187-3-1-8, ocupó el primer lugar en rendimiento en los ambientes de CEIXTA, Ver., en otoñoinvierno de 2016-17, así como en el ITA-JRC, Veracruz, en el mismo ciclo agrícola, en suelo ácido, con y sin aplicación de cal dolomita, en donde fue el de mayor eficiencia productiva (Tosquy et al., 2018). En tanto que, Jamapa Plus/XRAV-187-3-1-2 se ubicó en primer lugar en los ambientes de Nuevo México, Chiapas, en verano de 2016, en suelo ácido encalado, y en El Rubí, Veracruz, en invierno-primavera de 2017, con suspensión de riego al inicio de la etapa reproductiva del cultivo, en donde mostró mayor tolerancia a la sequía terminal y eficiencia productiva (con riego y sequía), que las variedades Negro Comapa y Negro Grijalva (Ibarra et al., 2018).

\section{Análisis AMMI}

De acuerdo al análisis AMMI, se detectó variabilidad altamente significativa en los primeros cinco componentes principales, los cuales acumularon $96.1 \%$ en la explicación de la varianza, de estos cinco, los primeros tres fueron los más importantes en la representación de la IGA, ya que explicaron $81.9 \%$ de la suma de cuadrados. Según Pereira et al. (2009), los primeros tres componentes principales de este análisis deben explicar más del 60\%, para considerarlo suficiente $\mathrm{y}$ al menos $70 \%$ como satisfactorio.

Este modelo permitió identificar tres grupos de ambientes relativamente homogéneos y bien definidos, en función de su rendimiento promedio y de la interacción de éstos con los genotipos (Williams et al., 2010). El primer grupo incluyó tres ambientes de Veracruz: El CEIXTA (A8), Rincón Grande (A4) y El Rubí (A5), todos ellos son similares en ciclo de cultivo (OI de 2016-17) 
y condición de humedad (humedad residual con distribución adecuada de lluvias). Estos ambientes combinaron alto rendimiento promedio de grano, significativamente superiores a la media general que fue de $1312 \mathrm{~kg} \mathrm{ha}^{-1} \mathrm{y}$ baja interacción con los genotipos (CP 1= -6.4547, -7.9732 y -11.2433) (Cuadro 3, Figura 1). Estas dos características, en el proceso de mejoramiento genético, los hacen ideales para identificar germoplasma de frijol negro opaco, con alto y estable rendimiento, para las zonas norte y región de Las altas Montañas en el centro del estado de Veracruz (López et al., 2015).

Cuadro 3. Rendimiento promedio de genotipos, ambientes y valores de componentes principales significativos.

\begin{tabular}{|c|c|c|c|c|c|}
\hline Tipo & Genotipo/ambiente & $\begin{array}{l}\text { Rendimiento } \\
\left(\mathrm{kg} \mathrm{ha}^{-1}\right)\end{array}$ & $\mathrm{CP} 1$ & $\mathrm{CP} 2$ & CP3 \\
\hline G1 & Papaloapan/SEN 46-3-7 & 1333.5 & 8.6001 & -20.7015 & 0.9565 \\
\hline G10 & Jamapa Plus/XRAV-187-3-1-8 & 1437.3 & 0.2246 & 16.4036 & -2.3564 \\
\hline G11 & Jamapa Plus/XRAV-187-3-1-2 & 1504.3 & 22.3616 & 2.5865 & 4.6737 \\
\hline G12 & Jamapa PlusX/RAV-187-3-4-4 & 1359.3 & -9.0003 & 7.5340 & -10.4285 \\
\hline G13 & Negro Comapa (TR) & 1446.2 & 9.7237 & 12.5242 & -2.7705 \\
\hline G14 & Negro Grijalva (TR) & 1471 & 15.4341 & 10.8310 & -3.9939 \\
\hline $\mathrm{G} 2$ & Papaloapan/SEN 46-6-6 & 1162.2 & -2.3531 & -16.2314 & -11.4931 \\
\hline G3 & Papaloapan/SEN 46-7-7 & 1202.8 & 1.0064 & -13.0445 & 12.9939 \\
\hline G4 & Papaloapan/SEN 46-7-11 & 1246.4 & -19.757 & -0.7866 & -7.2652 \\
\hline G5 & N Citlali/XRAV-187-3-1-6 & 1302.6 & -0.4576 & 2.8631 & 13.2464 \\
\hline G6 & N Citlali/XRAV-187-3-1-8 & 1377.5 & -12.615 & 5.0874 & 8.9055 \\
\hline G7 & N Citlali/XRAV-187-3-14-6 & 1171.6 & -5.1128 & -9.7054 & 1.7015 \\
\hline G8 & N Citlali/XRAV-187-3-14-7 & 1221.7 & -13.7884 & 7.4020 & 7.6905 \\
\hline G9 & N Citlali/XRAV-187-3-16-7 & 1144.9 & 5.7337 & -4.7624 & -11.8601 \\
\hline A1 & $\begin{array}{l}\text { Nuevo México, Villaflores, Chis., V 2016, T, suelo } \\
\text { ácido - CD }\end{array}$ & 1678.5 & 16.5182 & -11.5001 & 22.2422 \\
\hline A10 & El Rubí, Medellín, Ver., IP 2017, sequía terminal & 1002.5 & 15.7745 & 6.2012 & 0.7924 \\
\hline $\mathrm{A} 2$ & $\begin{array}{l}\text { Nuevo México, Villaflores, Chis., V 2016, T, suelo } \\
\text { ácido }\end{array}$ & 1070.8 & 9.2583 & 0.7911 & -15.364 \\
\hline A3 & Carranza, Ocozocoautla, Chis., OI 2016-17, HR & 1982.8 & -21.6850 & 24.4266 & 5.4789 \\
\hline A4 & Rincón Grande, Orizaba, Ver., OI 2016-17, HR & 1589.2 & -7.9732 & -23.1999 & -11.4617 \\
\hline A5 & El Rubí, Medellín, Ver., OI 2016-17, HR & 1494.3 & -11.2433 & 0.8056 & 4.289 \\
\hline A6 & ITA-JRC, Ver., OI 2016-17, HR, suelo ácido-CD & 667.6 & -7.0106 & -5.2081 & 1.0571 \\
\hline A7 & ITA-JRC, Ver., OI 2016-17, HR, suelo ácido & 480.9 & -6.8706 & -4.7559 & -0.312 \\
\hline A8 & CEIXTA, Tlapacoyan, Ver., OI 2016-17, HR & 1636.7 & -6.4547 & -4.763 & 0.9338 \\
\hline \multirow[t]{2}{*}{ A9 } & El Rubí, Medellín, Ver., IP 2017, riego & 1526.4 & 19.6864 & 17.2024 & -7.6558 \\
\hline & Media & 1312.9 & & & \\
\hline
\end{tabular}

$\mathrm{CP}=$ componente principal. $\mathrm{G}=$ genotipo. $\mathrm{A}=$ ambiente $\mathrm{V}=$ ciclo de verano. $\mathrm{OI}=$ ciclo de otoño-invierno. $\mathrm{IP}=$ ciclo de invierno- primavera. $\mathrm{T}=$ condición de temporal, $\mathrm{HR}=$ condición de humedad residual. $\mathrm{CD}=$ con aplicación de dolomita. ITA-JRC= Instituto Tecnológico Agropecuario de Juan Rodríguez Clara. CEIXTA= Campo Experimental Ixtacuaco. 
El segundo grupo incluyó dos ambientes en el ITA-JRC, Ver., similares en ciclo de cultivo (OI 2016-17) y condición de humedad (humedad residual con sequía terminal) y diferentes en el manejo del suelo: uno bajo condiciones de suelo ácido, sin aplicación de cal dolomita (A7) y otro con aplicación de cal dolomita (A6); ambos ambientes también mostraron reducida interacción con los genotipos (CP 1= -6.8706 y -7.0106, respectivamente), pero bajo rendimiento promedio, muy inferiores a la media general (Cuadro 3, Figura 1). Este comportamiento observado en ambos ambientes se atribuye principalmente, a la severidad del estrés hídrico que sufrieron los genotipos, lo cual comúnmente ocurre en esa zona productora de frijol (Morales et al., 2015), que no permitió que éstos expresaran su potencial de rendimiento y que las diferencias entre la gran mayoría de ellos fueran mínimas.

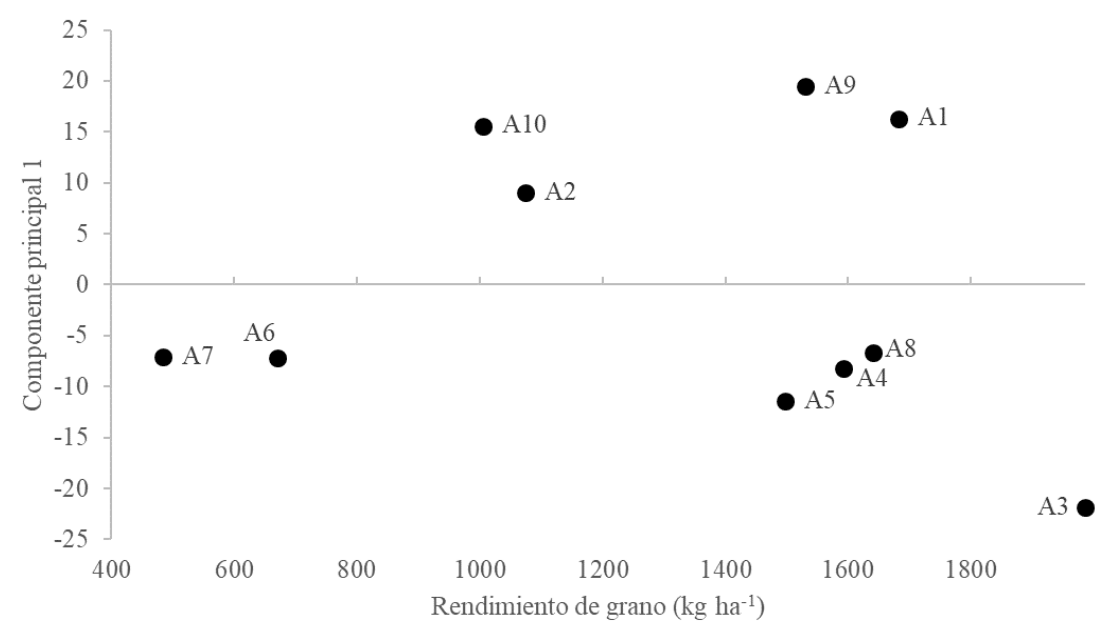

Figura 1. Efectos principales e interacción observada para 10 ambientes de prueba. A1= Nuevo México, Villaflores, Chiapas, con dolomita; A2= Nuevo México, Villaflores, Chiapas, sin dolomita; A3= Carranza, Ocozocoautla, Chiapas; A4= Rincón Grande, Orizaba, Veracruz; A5= El Rubí, Medellín, Veracruz; A6= Rodríguez Clara, Veracruz, con dolomita; A7= Rodríguez Clara, Veracruz, sin dolomita; A8= CEIXTA, Tlapacoyan, Veracruz; A9= El Rubí, Medellín, Veracruz, riego; A10= El Rubí, Medellín, Veracruz, sequía.

El tercer grupo incluyó tres ambientes con adecuada humedad durante el ciclo del cultivo: Carranza, Chis., ciclo de OI de 2016-2017, con humedad residual (A3), El Rubí, Ver., ciclo de IP de 2017, con riego durante el ciclo del cultivo (A9) y Nuevo México, Chis., en verano de 2016, bajo condiciones de temporal y de suelo ácido encalado con dolomita (A1), en los cuales se observó alto rendimiento promedio y la interacción más alta entre el ambiente y los genotipos $(\mathrm{CP} 1=-$ 21.685, 19.6864 y 16.5182, respectivamente) (Cuadro 3, Figura 1), esto obedece principalmente a diferencias en el potencial de rendimiento y adaptación de los genotipos en esos ambientes de evaluación, en los que se dispuso de adecuada humedad durante el desarrollo del cultivo.

Cabe resaltar, que estos tres ambientes de alto rendimiento promedio, que se encuentran lejos del eje de las ordenadas (Figura 1) y que contribuyeron más a la interacción genotipo-ambiente, en determinados casos, son adecuados para la producción de frijol, si se dispone de un genotipo con adaptación específica a esos ambientes (Acosta et al., 2012). 
En relación con los genotipos, sobresalieron dos grupos: uno conformado con materiales que mostraron muy baja interacción (cercana a cero) y por lo tanto, un comportamiento estable (López et al., 2011), en el que se incluye a la línea Jamapa Plus/XRAV-187-3-1-8 (G10), la cual además de haber obtenido alto rendimiento promedio, mostró la menor interacción con el ambiente con un CP 1 = 0.2246 (Cuadro 3 y Figura 2), así como a las líneas Negro Citlali/XRAV-187-3-1-6 (G5) y Papaloapan/SEN 46-7-7 (G3), que también presentaron reducida interacción con el ambiente (CP $1=-0.4576$ y 1.0064 , respectivamente), pero su rendimiento promedio fue más bajo (Figura 2), estos genotipos pueden utilizarse en los programas de mejoramiento genético, para la generación de líneas y variedades de frijol negro opaco con amplia adaptación. En la clasificación de genotipos por su estabilidad, utilizando el modelo AMMI, deben seleccionarse preferentemente los que se encuentren próximos al eje de las ordenadas, con valores del CP 1 igual o próximos a cero (que son los que interaccionan en menor grado con el ambiente) y muestren alto rendimiento de grano (Vargas y Crossa 2000; Pereira et al., 2009).

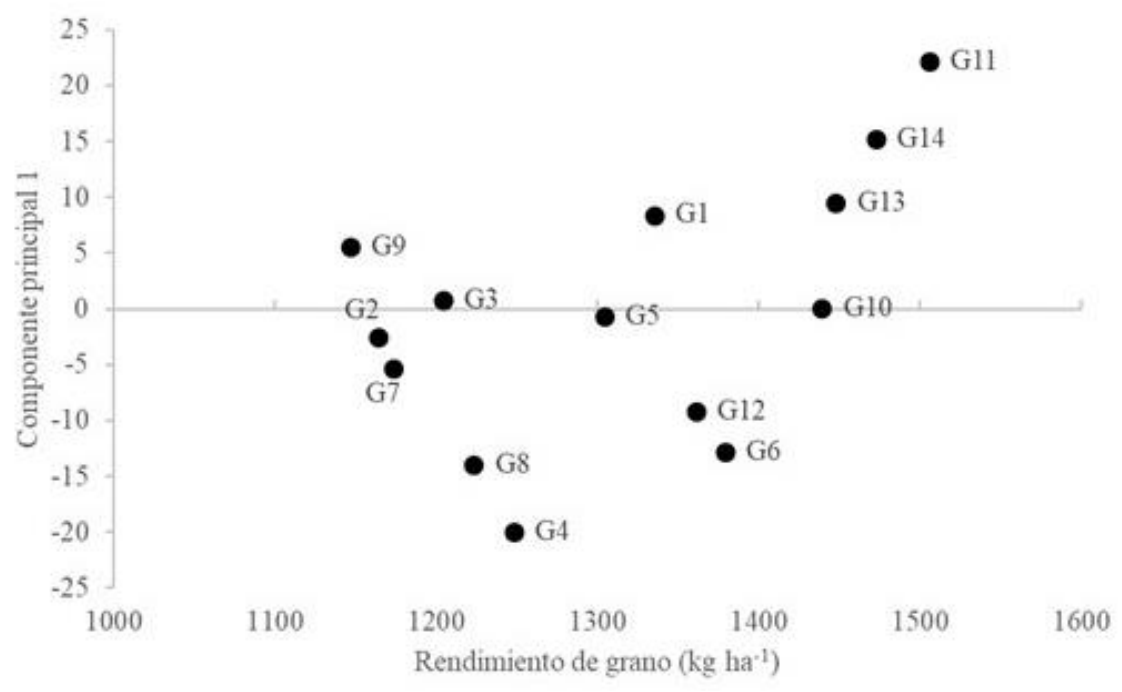

Figura 2. Efectos principales e interacción observada del rendimiento de 14 genotipos de frijol negro.

El otro grupo se conformó de tres genotipos que presentaron alto rendimiento promedio pero alta interacción con el ambiente; este grupo incluye la línea Jamapa Plus/XRAV-187-3-1-2 (G11), que fue la más productiva y la que interaccionó más con el ambiente (CP 1=22.33616) (Cuadro 3, Figura 2). Esta línea mostró adaptación específica en ambientes, con y sin estreses abióticos: por suelo ácido, en Nuevo México, Chiapas., y sequía terminal, en El Rubí, Veracruz, condiciones en las que obtuvo rendimientos de grano significativamente sobresalientes, por lo que en un futuro, puede representar una opción para su siembra en ese tipo de suelos en el centro del estado de Chiapas y en otoño-invierno, bajo condiciones de humedad residual, en el estado de Veracruz, en donde es común la ocurrencia de sequía durante la etapa reproductiva del cultivo (Tosquy et al., 2014). Los otros dos genotipos fueron: Negro Grijalva (G14) con CP 1=15.4341 y Negro Comapa (G13) con CP 1= 9.7237 (Cuadro 3, Figura 2), estas dos variedades mostraron adaptación específica a las condiciones de riego y sequía terminal en El Rubí, Veracruz, y de acidez edáfica en Nuevo México, Chiapas; Negro Comapa también mostró una respuesta significativa en el rendimiento de grano, a la aplicación de cal dolomita, en este último ambiente. La adaptación específica en un área determinada también es deseable, si el genotipo presenta estabilidad a través de años en esa área y alto rendimiento de grano (Acosta et al., 2012). 
Estos resultados indican que, la estabilidad del rendimiento de los genotipos evaluados no está en función del acervo genético de donde provienen, ya que en general, materiales que se derivaron de una misma cruza, mostraron diferencias en su potencial de rendimiento y adaptación a los ambientes de prueba.

\section{Conclusiones}

El análisis AMMI permitió identificar genotipos con base a su nivel de interacción con el ambiente y rendimiento de grano. La línea Jamapa Plus/XRAV-187-3-1-8 (G10), fue la que mostró la mayor estabilidad en los ambientes de evaluación (superior a la observada por las variedades Negro Comapa y Negro Grijalva), así como alto rendimiento de grano, y por ello se incluirá en el proceso de validación con agricultores cooperantes en los estados de Veracruz y Chiapas. En tanto que, la línea Jamapa Plus/XRAV-187-3-1-2 (G11), fue la de mayor rendimiento promedio y su adaptación fue específica, por lo que debe considerarse su utilización en los ambientes en los que obtuvo rendimientos de grano significativamente sobresalientes, principalmente bajo condiciones de suelo ácido en el centro del estado de Chiapas y de sequía terminal en el centro del estado de Veracruz.

\section{Literatura citada}

Acosta, D. E.; Acosta, G. J. A.; Trejo, L. C.; Padilla, R. J. S. and Amador, R. M. D. 2009. Adaptation traits in dry bean cultivars grown under drought stress. Agric. Téc. Méx. 35(4):416-425.

Acosta, G. J. A.; Espinoza, T. E.; Sánchez, G. B. M.; Jiménez, H. Y.; Salinas, P. R. A.; Rosales, R. S. R.; Zandate, H. R. y González, R. C. 2012. Adaptación del frijol tipo Flor de Junio a diferentes ambientes de México. Trop. Subtrop. Agroecosyst. 15(2):427-438.

Arias, R. J. H.; Rengifo, M. T. y Jaramillo, C. M. 2007. Manual técnico: buenas prácticas agrícolas en la producción de fríjol voluble. $1^{\mathrm{a}}$. ed. Corporación Colombiana de Investigación Agropecuaria (CORPOICA). Mejoramiento Alimentario y Nutricional de Antioquia (MANA). Organización de las Naciones Unidas para la Agricultura y la Alimentación (FAO). Medellin, Colombia. 170 p.

Brancourt-Hulmel, M. and Lecomte, C. 2003. Effect of environmental variates on genotype environment interaction of winter wheat: a comparison of biadditive factorial regression to AMMI. Crop Sci. 43(2):608-617.

Crossa, J.; Gauch, Jr., H. G. and Zobel, R. W. 1990. Additive main effects and multiplicative interaction analysis of two international maize cultivar trails. Crop Sci. 30(3):493-500. Eberhart, S. A. and Russell, W. A. 1966. Stability parameters for comparing varieties. Crop Sci. 6(1):36-40.

FIRA. 2016. Fideicomiso Instituido con Relación a la Agricultura. Panorama agroalimentario. Dirección de Investigación y Evaluación Económica y Sectorial. Frijol 2016. FIRA. México. 36 p.

Gauch Jr., H. G. and Furnas, R. E. 1991. Statistical analysis of yield trails with MATMODEL. Agron. J. 83(5):916-920.

Gauch, H. G. and Zobel, R. W. 1996. AMMI analysis of yield trials. In: Kang, M. S. and Gauch, H. G. (eds.). Genotype-by-environment interaction. CRC Press. Boca Raton, FL, USA. 85$122 \mathrm{pp}$. 
Ibarra, P. F. J.; Tosquy, V. O. H.; López, S. E.; Rodríguez, R. R.; Villar, S. B.; Anaya, L. J. L.; Garrido, R. E. and Zetina, L. R. 2018. Promising black-seeded common bean breeding lines resistant to $\mathrm{BCMV}, \mathrm{BCMNV}$ and $\mathrm{BGYMV}$ with adaptation to terminal drought and acid soils of tropical southeastern Mexico. The LXI Ann. Rep. Bean Improv. Coop. 65-66 pp.

López, S. E.; Acosta, G. J. A.; Tosquy, V. O. H.; Salinas, P. R. A.; Sánchez, G. B. M.; Rosales, S. R.; González, R. C.; Moreno, G. T.; Villar, S. B.; Cortinas, E. H. M. y Zandate, H. R. 2011. Estabilidad de rendimiento en genotipos mesoamericanos de frijol de grano negro en México. Rev. Mex. Cienc. Agríc. 2(1):29-40.

López, S. E.; Tosquy, V. O. H.; Jiménez, H. Y.; Salinas, P. R. A.; Villar, S. B. y Acosta, G. J. A. 2012. Rendimiento y adaptación de la variedad de frijol Negro Comapa en dos regiones de México. Rev. Fitotec. Mex. 35(4):309-315.

López, S. E.; Tosquy, V. O. H.; Villar, S. B.; Acosta, G. J. A.; Rodríguez, R. J. R. y Meza, P. A. 2015. Rendimiento y estabilidad de líneas mejoradas de frijol negro en Veracruz y Chiapas, México. Rev. Fitotec. Mex. 38(2):173-181.

Morales, R. A.; López, C. C.; Kohashi, S. J.; Miranda, C. S. y García, E. A. 2015. Comparación de los componentes del rendimiento en variedades de frijol en condiciones de acidez y humedad residual del suelo en el sur de Veracruz. Terra Latinoam. 33(4):309-319.

Pereira, H. S.; Melo, L. C.; de Faria, L. C.; Díaz. J. L. C.; Del Peloso, M. J.; da Costa, J. G. C. and Wendland, A. 2009. Stability and adaptability of carioca common bean genotypes in states of the central South Region of Brazil. Crop Breed. Appl. Biotechnol. 9(2):181-188.

Rodríguez, L. G.; García, S. J. A.; Rebollar, R. S. y Cruz, C. A. C. 2010. Preferencias del consumidor de frijol (Phaseolus vulgaris L.) en México: factores y características que influyen en la decisión de compra diferenciada por tipo y variedad. Paradigma Económico. 2(1):121-145.

Ruiz, C. J. A.; Medina, G. G.; González, A. I. J.; Flores, L. H. E.; Ramírez, O. G.; Ortiz, T. C.; Byerly, M. K. F. y Martínez, P. R. A. 2013. Requerimientos agroecológicos de cultivos. $3^{\text {a }}$. (Ed.). INIFAP. CIRPAC. Campo Experimental Centro Altos de Jalisco. Tepatitlán de Morelos, Jal., México. Libro técnico Núm. 3.564 p.

SAS. 1999. Institute Statistical Analysis Systems. SAS/STAT user's guide. Software versión 8.0. SAS Institute Inc. Cary, NC, USA.

SIAP. 2018. Servicio de Información Agroalimentaria y Pesquera. Avances de siembras y cosechas. Resumen por cultivo. SAGARPA. SIAP. Ciudad de México, México. http://infosiap.siap.gob.mx:8080/agricola_siap_gobmx/ResumenDelegacion.do.

Singh, S. P. 1982. A key for identification of different growth habits of Phaseolus vulgaris L. The XXV Ann. Rep. Bean Improv. Coop. 92-95 pp.

Tosquy, V. O. H.; López, S. E.; Esqueda, E. V. A.; Acosta, G. J. A.; Ugalde, A. F. J. y Villar, S. B. 2012. Rendimiento y reacción a enfermedades de genotipos de frijol en condiciones de temporal y humedad residual. Rev. Mex. Cienc. Agríc. 3(4):727-737.

Tosquy, V. O. H.; López, S. E.; Francisco, N. N.; Acosta, G. J. A y Villar, S. B. 2014. Genotipos de frijol negro opaco resistentes a sequía terminal. Rev. Mex. Cienc. Agríc. 5(7):12051217.

Tosquy, V. O. H.; López, S. E.; Zetina, L. R.; Ugalde, A. F. J.; Villar, S. B. y Cumpián, G. J. 2008. Selección de genotipos de frijol con adaptación a suelos ácidos. Terra Latinoam. 26(3):227-233. 
Tosquy, V. O. H.; López, S. E.; Zetina, L. R.; Villar, S. B. y Rodríguez, R. J. R. 2017. Producción de genotipos de frijol negro en condiciones de humedad residual y sequía terminal. Terra Latinoam. 35(1):29-39.

Tosquy, V. O. H.; Zetina, L. R.; Ibarra, P. F. J. and López, S. E. 2018. Improved opaque black common bean gentopyes with adaptation to acid soils. The LXI Ann. Rep. Bean Improv. Coop. 207-208 pp.

Vallejo, C. F. A. 2005. Análisis estadístico para los diseños genéticos en fitomejoramiento. 1ª . ed. Universidad Nacional de Colombia. Palmira, Colombia. 249 p.

Vargas, E. E. A.; Vargas, S. J. E. y Baena, G. D. 2016. Análisis de estabilidad y adaptabilidad de híbridos de maíz de alta calidad proteica en diferentes zonas Agroecológicas de Colombia. Acta Agron. 65(1):72-79. doi: https://doi.org/10.15446/acag.v65n1.43417.

Vargas, H. M. y Crossa, J. 2000. El análisis AMMI y la gráfica del biplot en SAS. 1a . (Ed.). Centro Internacional para el Mejoramiento de Maíz y Trigo (CIMMYT). México, D. F. 42 p.

Villar, S. B.; López, S. E. y Acosta, G. J. A. 2003. Selección de genotipos de frijol por rendimiento y resistencia al mosaico dorado y suelos ácidos. Rev. Fitotec. Mex. 26(2):109-114.

Villar, S. B.; López, S. E. y Tosquy, V. O. H. 2009. Negro Grijalva, nuevo cultivar de frijol para el trópico húmedo de México. Agric. Téc. Méx. 35(3):349-352.

Williams, A. H.; Pecina, Q. V.; Zavala, G. F.; Montes, G. N.; Gámez, V. A. J.; Arcos, C. G.; García, G. M. A.; Montes, H. S. y Alcalá, S. L. 2010. Modelo de Finlay y Wilkinson vs. el modelo AMMI para analizar la interacción genotipo-ambiente en sorgo. Rev. Fitotec. Mex. 33(2):117-123. 